\title{
La investigación científica en el proceso de aprendizaje para la enseñanza: educación, sociedad y ciencia
}

\author{
Wendy Villafranqui Cabanillas \\ Universidad de Buenos Aires, CA.B.A., Argentina \\ https://orcid.org/0000-0003-2685-6643
}

Recibido:06/10/20 Revisado: 06/11/20 Aceptado: 18/11/20 Publicado: 05/12/2020

\begin{abstract}
Resumen
La situación de pandemia mundial ha puesto en evidencia que el consumo de noticias falsas, la predilección por artículos de difusión de la ciencia antes que los artículos de investigación científica y la legitimación de las redes sociales como fuentes de información afectan el desarrollo social y mantienen a los involucrados vulnerables, tanto como susceptibles a la desinformación. Así, la necesidad de formar ciudadanos con perfil investigador, interesados por la ciencia y familiarizados con sus discursos resulta imperiosa. En el presente trabajo se pretende, mediante una investigación documentada que engloba estudios sociales, enfoques pedagógicos y análisis discursivos, reflexionar acerca de la relación entre la situación actual del discurso científico con la educación y, en consecuencia, su repercusión en el desarrollo de la sociedad. Al finalizar, se propone un método de trabajo para las entidades de formación docente focalizado en la interacción con textos de investigación científica.

Palabras clave: investigación; ciencia y desarrollo; educación y desarrollo; pandemia
\end{abstract}

\section{Scientific research in the learning process for teaching: education, society and science}

\begin{abstract}
The global pandemic situation has made it clear that the consumption of fake news, the predilection for articles to disseminate science rather than scientific research articles, and the legitimation of social networks as sources of information affect social development and maintain those involved vulnerable, as well as susceptible to misinformation. Thus, the need to train citizens with a research profile, interested in science and familiar with its discourses is imminent. In this paper, through documented research that includes social studies, pedagogical approaches and discursive analysis, it's intended to reflect on the relationship between the current situation of scientific discourse and education, as consequently, its impact on the development of the society. At the end, a working method is proposed for teacher training entities focused on interaction with scientific research texts.

Keywords: scientific research; science and development; education and development; pandemics
\end{abstract}

\section{A pesquisa científica no processo de aprendizagem para o ensino: educação, sociedade e ciência}

Resumo

A situação de pandemia global deixou claro o consumo de notícias falsas, preferindo artigos de divulgação científica, ao em vez de artigos de pesquisa científica e a legitimação das redes sociais como fontes de informação afetam o desenvolvimento 
social e mantém os envolvidos vulneráveis, bem como suscetíveis a desinformaçã. Gerando assim, a necessidade de formar cidadãos com perfil de pesquisador, interessados em ciência e familiarizá-los com seus discursos é iminente. Neste artigo, por meio de pesquisas que incluem estudos sociais, abordagens pedagógicas e análise discursiva, pretende-se refletir sobre a relação entre a situação atual da ciência, discurso e educação, e consequentemente, seu impacto no desenvolvimento da sociedade. Ao final, é proposto uma metodologia de trabalho para entidades formadoras de professores voltados para interação com textos de pesquisa científica.

Palavras-chave: investigação; ciencia e desenvolvimento; educação e desenvolvimento; pandemia

Citar como:

Villafranqui, W. (2020). La investigación científica en el proceso de aprendizaje para la enseñanza: educación, sociedad y ciencia. Revista Digital de Investigación en Docencia Universitaria. 14(2), e1359. https://doi.org/ 10.19083/ ridu.2020.1359

\section{Antecedentes}

Nuestra sociedad se encuentra enfrentando una situación que pone a prueba no solo la estabilidad de nuestras estructuras sociales, sino también las competencias de los ciudadanos que la conforman. La creciente popularidad de las noticias falsas, el mayor consumo de artículos de difusión de la ciencia (adaptados y parcializados) antes que artículos de investigación científica (detallados y metódicamente estructurados) y la legitimación de las redes sociales como fuentes de información que superan en suscriptores locales a las revistas científicas nacionales e internacionales están afectando considerablemente el desarrollo social, indisponiendo a los ciudadanos en su búsqueda de una mejor calidad de vida y disminuyendo su expectativa de superación ante la situación de pandemia mundial.

Tomando ello en consideración y teniendo como referentes las investigaciones de González (2019) y Marmón (2014) que explican problemas sociales relacionados al discurso científico, el crecimiento de las fake news o noticias falsas, así como su consumo y el desconocimiento de los filtros informativos; además del estudio de Muñoz, Muñoz, García y Granado (2013) que relaciona los discursos de la ciencia con su aplicación en el ámbito educativo, se ha identificado al manejo de información de nuestra sociedad como una debilidad cuya mejora debe priorizarse. Para poder abordarla se ha considera- do como premisa la afirmación que declara Bajtín (1982): solo podemos desenvolvernos en aquellos géneros discursivos con los que hemos aprendido a interactuar, es justamente esta interacción la que nos faculta su dominio.

Los estudios de García (2008), Ciapuscio (2011), y Llácer y Ballesteros (2012) respecto de los recursos lingüísticos utilizados en el discurso científico, contraponiendo el artículo de investigación al artículo de difusión de la ciencia, se tomarán como base para, a modo de sugerencia de trabajo para las entidades de formación docente, focalizar la interacción con textos de investigación científica.

En el presente artículo se expondrá una reflexión acerca de la situación de la sociedad actual y su relación con el discurso científico, así como la presencia (o ausencia) del mismo en los manuales escolares y exámenes de ingreso a una educación superior estatal. En el último apartado, se detallará una propuesta de trabajo dirigida a estudiantes de formación docente apelando al receptor final de este proceso: el ciudadano.

\section{Discusión}

\section{Situación Social Relacionada al Discurso Científico}

La sociedad peruana se encuentra en una situación en la que el manejo de información adecuada 
resulta tan importante como determinante para la supervivencia. En la primera mitad de 2020, las fuentes digitales, por seguridad, se convirtieron en fuentes únicas de información y con ello surgieron exigencias para poder adaptarse de manera competente a esta nueva interacción con los avances científicos.

Las fake news o noticias falsas se han convertido en un obstáculo significativo a la hora de discernir información. Según González (2019), “durante 2017 se detectaron 122 páginas webs dedicadas a publicar noticias falsas" (p. 33). Contra ellas se han creado páginas como "Fact Check" (de Google) y Maldito Bulo (de maldita.es) que permiten identificarlas y descartar las fuentes. Pese a ello, medios de comunicación nacionales han sido víctimas en más de una ocasión del engaño, con lo que gran parte de la población se ha visto afectada. Además, debido a que representantes nacionales o líderes de Estado legitiman las plataformas como Twitter y Facebook publicando comunicados, anuncios y opiniones en ellas, las noticias falsas cobran dimensión de rumores y se distribuyen en anonimato haciendo cada vez más difícil borrar su rastro.

De acuerdo con González (2019), "las redes sociales han introducido el concepto de autodistribución, el cual implica que son los propios usuarios quienes deciden a través de qué canales iniciar la difusión de un contenido" (p.34).

Las noticias falsas suelen imitar el discurso de difusión de la ciencia y se propagan en medios sin supervisión (como las redes sociales), mediante descripciones con elementos adjuntos o enlaces que dirigen a páginas dudosas o poco conocidas, pero a las que la población más vulnerable se encuentra mayormente expuesta; la preferencia por el artículo de difusión se debe a que el discurso de investigación científica es difícil de imitar debido a la cantidad de intertextos ${ }^{1}$ (citas, alusiones, transposiciones, etc.), además del lenguaje preciso y objetivo que no apela a la sensibilidad del lector, sino a su racionalidad.

Se puede, por lo tanto, deducir que el dominio del discurso de investigación científica por la población podría disminuir el consumo de noticias

1 El término ha sido tomado de los estudios de interdiscurso realizados por Adam (1999) falsas y su propagación. El conocimiento y dominio de fuentes fidedignas nacionales e internacionales para el acceso a información clasificada se presenta como necesario en cualquier momento de la historia y es una competencia que todo ciudadano debería adquirir.

\section{El Discurso de Investigación Científica y la Educación}

La clasificación y determinación de los géneros discursivos son producto aún de estudios inconclusos; sin embargo, la certeza de que podemos desenvolvernos solo en aquellos que, generalmente de manera inconsciente, conocemos y dominamos por práctica o interacción es irrefutable. Bajtín (1982) explica que, si se logra dominar un género discursivo específico, el enunciador podrá interactuar con este con mayor libertad según su intención enunciativa. Se deduce, por lo tanto, que la falta de interés por los textos científicos o participación en debates de la misma índole se debe a una inhabilidad para dominar los géneros correspondientes al discurso científico y esta dificultad, a la poca interacción que se ha tenido con el género durante la formación del individuo.

Prueba de ello es la presencia casi monótona de textos breves, fragmentados o resumidos en los manuales escolares (dejando de lado el plan lector que, en su aplicación, solo incluye al género literario, pese a que el Ministerio de Educación (2006) sugiere diversidad en la selección de textos) ${ }^{2}$; la predilección por discursos periodísticos y artículos de difusión de la ciencia en el examen de admisión de la universidad con mayor cantidad y diversidad de postulantes (Universidad Nacional Mayor de San Marcos, UNMSM), lo que llevaría también a repensar el perfil deseado de estudiante universitario; y la producción de artículos de investigación que, en Latinoamérica, ubica a las universidades nacionales peruanas a partir del puesto 45 (Scimago Institutions Rankings, 2020).

Tomando como ejemplo los textos utilizados para evaluar la comprensión lectora en los últimos cinco exámenes de admisión a la UNMSM e identi-

2 Se puede verificar revisando las páginas de las editoriales a cargo como Santillana (programa Loqueleo) o Black Cat (para el plan lector en inglés). 
ficando sus fuentes, se ha elaborado la siguiente tabla (ver Tabla 1).

Como se puede observar, de 21 textos seleccionados para evaluar a los ingresantes de las áreas de Ingeniería, Humanidades, y Ciencias Jurídicas y Sociales, solo 6 corresponden a investigaciones científicas relacionadas con dichas áreas.

Si bien los géneros condensados en la categoría "otros" de la Tabla I corresponden a fuentes diversas, tales como periódicos nacionales (El Comercio, La República) e internacionales (El País), blogs, boletines informativos (National Geographic) y manuales escolares (tomados a partir del proceso 2019-I, debido a la introducción de textos en inglés), se resalta la relación directa de su contenido con las diferentes especialidades agrupadas en cada área.

De los artículos de investigación científica, se reconocen fuentes que pueden ser clasificadas entre indexadas y no indexadas (ver Tabla 2); asimis- mo, muchos de ellos, por longitud y para fines de condensación de información que se corresponda con el tiempo dedicado a cada texto en el examen de admisión han sido adaptados.

Cabe destacar que la indización es una herramienta que facilita la búsqueda a público diverso, profesionales o estudiantes, mas no debe tomarse como un factor para desestimar revistas nacionales que no cuentan con dicha distinción, ya que eso no disminuye la calidad del trabajo del investigador (téngase en cuenta que muchas revistas no indexadas cuentan con publicaciones en revistas que sí lo están).

En la actualidad se dispone de estudios que han demostrado la importancia de los discursos de la ciencia para la adquisición de competencias y desarrollo de habilidades comunicativas. En Muñoz et al. (2013), se describen los aportes de los textos científicos basados en las características que des-

Tabla 1

Clasificación de Textos Seleccionados para los Últimos Cinco Procesos de Admisión a la UNMSM Según sus Fuentes

\begin{tabular}{|c|c|c|c|}
\hline \multirow{2}{*}{ Proceso de admisión } & \multicolumn{2}{|c|}{ Géneros } & \multirow{2}{*}{ Total de textos } \\
\cline { 2 - 4 } & $\begin{array}{c}\text { Artículo de investigación } \\
\text { científica }\end{array}$ & Otros & 4 \\
\hline $2020-\mathrm{I}$ & 2 & 2 & 4 \\
\hline 2019-II & 1 & 4 & 5 \\
\hline 2019-I & 1 & 2 & 4 \\
\hline 2018-II & 2 & 4 & 4 \\
\hline 2018-I & 0 & 15 & 21 \\
\hline Total & 6 & & 2 \\
\hline
\end{tabular}

Tabla 2

Clasificación de Fuentes de los Artículos de Investigación Científica Según su Indexación

\begin{tabular}{|l|c|c|c|}
\hline Fuente & Indexada & No indexada & Nota \\
\hline Contextos & $\mathrm{X}$ & & \\
\hline $\begin{array}{l}\text { Ciencia, Tecnología y } \\
\text { Sustentabilidad }\end{array}$ & & $\mathrm{X}$ & Con publicaciones citadas en revistas indexadas \\
\hline Ideele & $\mathrm{X}$ & $\mathrm{X}$ & Con publicaciones citadas en revistas indexadas \\
\hline Revista de Medios y Educación & $\mathrm{X}$ & & \\
\hline Forma y Función & $\mathrm{X}$ & & \\
\hline Alteridades & & & \\
\hline
\end{tabular}


tacan su función en la actividad académica.

Reflejan la actividad intelectual del hombre, el progreso de la ciencia y la técnica. Tiene como características esenciales la impersonalidad, la objetividad y la exactitud. Generalmente, el texto científico está construido no solamente con un lenguaje, sino que incluye también otros sistemas de comunicación tales como: metalenguaje específico de la ciencia de que se trate, gráficos, fórmulas, etc. La función comunicativa actúa aquí en su forma informativa. (p.792)

A este aporte cabe agregar que un texto científico permite estimular el trabajo de búsqueda y revisión de fuentes con la finalidad de abordar las diferentes aristas de un problema, contrastar los criterios de diversos autores, llegar a conclusiones propias que reflejen su juicio y concebir proyectos en cuya aplicación la participación de un docente orientador, facilitador y crítico será primordial.

Un artículo de investigación científica, a diferencia de uno de difusión de la ciencia, estimula al lector a formar un juicio propio, debido a la imparcialidad con la que se presenta; expone, describe y argumenta haciendo uso activo del interdiscurso que mantendrá al estudiante en una dinámica donde el lenguaje se figura como completo, funcional y totalmente viviente; permite reconocer y replicar el uso de conectores lógicos y marcadores discursivos que facilitan la organización de ideas en cualquier campo de investigación e interactúan constantemente con la realidad, sea para explicar la actualidad, para presentarla o para exponer futuros cambios a través de análisis minuciosamente establecidos.

Visto desde otra perspectiva, familiarizarse con los discursos de la ciencia estimula el desarrollo de habilidades para interactuar en sociedad haciendo uso de los diferentes niveles de lenguaje; crea ciudadanos activos con criterio y autonomía; y fomenta la participación en la comunidad científica nacional e internacional, lo que fortalece a las instituciones y organizaciones que se dedican a la investigación cuyos resultados benefician a la población dándole prestigio y relevancia mundial, situación que permitirá luchar contra la minorización lingüística que viven las comunidades hispanohablantes, entre otras (Arnoux, 2014).

\section{Propuesta para la Aplicación del Discurso Científico en el Campo Pedagógico}

Como ser humano que incursiona en el arte de hablar, el desarrollo evolutivo del discurso científico al que se someterá a personas no relacionadas con sus géneros discursivos requerirá del pleno compromiso de sus mediadores; por ello, todo estudiante que se prepara para la docencia debe aprender a adaptar artículos de investigación científica a artículos de difusión de la ciencia. Esto permitirá al estudiante a docencia dominar el material de referencia, así como familiarizarse con las diversas fuentes. Es importante recordar que dicho proceso no consistirá en minorizar el discurso de investigación, por el contrario, consistirá en adaptarlo para poder focalizar su contenido de acuerdo a la finalidad pedagógica (que depende a su vez de las necesidades del contexto) para luego mostrarlo como la cumbre del trabajo, el ápex de la investigación.

Tomando como referencia la división por periodos con la que el Ministerio de Educación agrupa a los niveles educativos, una propuesta sería trabajar los artículos de difusión de la ciencia y sus referentes científicos hasta el periodo VI y los artículos de investigación científica en el periodo VII que abarca los últimos niveles académicos (4to y 5 to de secundaria). Sin embargo, en esta propuesta se sugiere que la exposición y dominio de ambos discursos se proponga en las Facultades de Educación, ya que toda innovación realizada en la formación de docentes tiene un rápido y eficaz efecto multiplicador en el rendimiento del sistema educativo.

Al respecto, como se mencionó anteriormente, al igual que para la adquisición de una lengua materna, los géneros discursivos se aprenden en la interacción con el género mismo, aunque no se tenga consciencia plena de ello, por lo que dotar a los futuros mediadores y, posteriormente, futuros científicos de este conocimiento solo se logrará exponiéndolos a él, acostumbrándolos al discurso científico (etapa pasiva) e incentivando su participación mediante estudios o proyectos para aplicación en las aulas (etapa activa). Para ello, la etapa de participación pasiva es el primer eslabón en la cadena de formación de investigadores. En esta etapa, la elaboración de un sílabo que incluya revisión de artículos de revistas científicas, parti- 
cipación en simposios y programación de debates de temas de actualidad por especialidades resulta, no solo apropiada, sino también necesaria.

Llácer y Ballesteros (2012) detallan las diferencias entre el lenguaje de artículos de investigación científica y el lenguaje de la divulgación de la ciencia, diferencia que puede ser utilizada como referente estructural de ambos discursos y como camino para lograr uno basado en el otro. (ver Tabla 3).

Esta adaptación permitirá captar la atención de los estudiantes a docencia y facilitará la asimilación de su labor como un compromiso de descubrimiento y aprendizaje constante. Con ello, además, adquirirán habilidades para transmitir a sus estudiantes y seguir la cadena de formación de investigadores activos.

Ciapuscio (2011), quien dedicó un artículo al estudio del discurso científico, mencionó que "la metáfora constituye un mecanismo de conceptualización de enorme importancia en el campo de la creación y la comunicación de la ciencia" (p. 91). Esta afirmación nos brinda un recurso de adaptación del discurso científico para fines pedagógicos de difusión, ya que la metáfora como tal, constituye un recurso comunicativo efectivo para la explicación y permite la construcción de interpretaciones adecuadas por parte de distintos tipos de audiencias. Debido a que evoca dominios cercanos a la experiencia cotidiana, resulta efectiva para abrir nuevos modos y caminos de pensamiento.

Otro mecanismo crucial para la elaboración de un artículo de difusión científica es las citas integradas y destacadas (García, 2008). Al interior del artículo final, facilitarán la comunicación intertextual y con ello la familiarización con sus referentes, además de brindar respaldo académico al autor. El manejo de fuentes bibliográficas durante el periodo de adiestramiento (etapa pasiva) será obligatorio para todos los involucrados, así como el conocimiento de las normas para autores detallados en los portales web de revistas científicas. Cada artículo de difusión propuesto deberá, a su vez, contar con el (o los) artículo de investigación científica tomado como referente adjunto para no perder de vista el objetivo final: formar investigadores activos.

Es preciso aclarar que el uso del artículo de difusión solo resultaría efectivo para los fines propuestos siempre y cuando se aplique en los primeros niveles de su educación formal (para la Educación Básica Regular, EBR), las adaptaciones mencionadas podrían extenderse hasta el primer grado del nivel VII; para la educación universita-

\section{Tabla 3}

Diferencias Entre los Recursos Estructurales y Lingüísticos del Artículo de Divulgación de la Ciencia y el Artículo de Investigación Científica

\begin{tabular}{|l|l|}
\hline \multicolumn{1}{|c|}{ Artículos de investigación científica } & \multicolumn{1}{|c|}{ Artículos de divulgación de la ciencia } \\
\hline $\begin{array}{l}\text { Presenta una estructura fija y obligatoria } \\
\text { - Introducción } \\
\text { - Materiales y métodos } \\
\text { - Conultados }\end{array}$ & $\begin{array}{l}\text { Presenta un resumen que refleja los puntos más } \\
\text { importantes de la investigación. }\end{array}$ \\
\hline $\begin{array}{l}\text { Títulos de gran extensión con exactitud y precisión del } \\
\text { tema por tratar. }\end{array}$ & $\begin{array}{l}\text { Títulos comprensibles para el lector medio, buscan atraer } \\
\text { la atención y guiarlo hacia un tema interesante. }\end{array}$ \\
\hline Presentan gráficos de líneas o barras y tablas. & $\begin{array}{l}\text { Muestran figuras, esquemas gráficos con el fin de resumir } \\
\text { y esquematizar visualmente la información. }\end{array}$ \\
\hline $\begin{array}{l}\text { Normativa que reproduce el proceso de la investigación } \\
\text { (planteamiento, experimentación y conclusión). }\end{array}$ & $\begin{array}{l}\text { Contienen componentes periodísticos y didácticos, } \\
\text { adapta la terminología, emplea recursos de tipo } \\
\text { metalingüístico (como cursiva, comillas, paréntesis), y } \\
\text { responde a una secuencia denominada "progresión lineal } \\
\text { simple" (de tema a rema) }\end{array}$ \\
\hline Lenguaje impersonalizado. & $\begin{array}{l}\text { El divulgador es protagonista y narra cronológicamente } \\
\text { (argumento, nudo y posible desenlace) }\end{array}$ \\
\hline
\end{tabular}


ria, formaría parte de los cursos generales (ciclos II y III) ${ }^{3}$. Al llegar a los límites mencionados, se deberá trabajar con artículos de investigación científica previamente seleccionados según el área de interés o el tema por desarrollar basados en proyectos establecidos (sugeridos por el docente o propuestos por el estudiante) prefijados al inicio del curso.

\section{Conclusiones}

Las fake news o noticias falsas, la falta de diversidad de géneros discursivos en los manuales escolares y la popularidad de los artículos de difusión de la ciencia antes que los de investigación científica ha mantenido a la población susceptible durante periodos críticos de la historia en donde el conocimiento de fuentes de información y el dominio de la misma marcan diferencias significativas.

Familiarizarse con los discursos de la ciencia, en especial con el artículo de investigación, resultará fructífero $\mathrm{y}$, a la larga, ventajoso para el desarrollo de toda sociedad, ya que abastecerá a sus ciudadanos de herramientas para luchar contra las noticias falsas, tendenciosas y los discursos parcializados ante los que actualmente se encuentran vulnerables.

Reconocer las características de los diversos géneros propuestos por la ciencia permite disponer de sus beneficios y hacerlos provechosos para la actividad pedagógica, por lo que no es un recurso que se deba dejar pasar

Los estudios de textos científicos han demostrado lo productivos que resultan en los distintos niveles de desarrollo de competencias lingüísticas y la mejor manera de propagar sus resultados es mediante su aplicación en las aulas de los estudiantes a docencia, quienes se encargarán de reproducir los resultados en la EBR.

3 La traducción de un género a otro requiere de la adquisición de competencias lingüísticas específicas. Por ello, resulta necesario reforzar el dominio de las funciones sintácticas, semánticas y pragmáticas en el ciclo I.

\section{Referencias}

Adam, J. (1999), Linguistique textuelle. Des genres de discours aux textes [Lingüística textual. De los géneros de discurso a los textos]. París, Francia: Nathan

Arnoux, E. (2014). Minorización lingüística y diversidad: en torno al español y al portugués como lenguas científicas. En J. Machado (Presidenta). As linguas portuguesa e española no cenário atual. Seminario Ibero-americano de diversidade lingüística. Instituto do Patrimonio Histórico e Artístico Nacional (IPHAN), Paraná. https://bit.ly/358SNq3

Bajtín, M. (1982). Estética de la creación verbal. Buenos Aires, Argentina: Siglo Veintiuno Editores S.A.

Ciapuscio, G. (2011). De metáforas durmientes, endurecidas y nómades: un enfoque lingüístico de las metáforas en la comunicación de la ciencia. ARBOR Ciencia, Pensamiento y Cultura. Volumen 187 (747), pp. 89 98. http://doi.org/10.3989/arbor.2011.747n1010

García, M. (2008). Subjetividad y discurso científicoacadémico. Acerca de algunas manifestaciones de la subjetividad en el artículo de investigación en español en el artículo de investigación en español. Revista Signos. Volumen 41 (66), pp. 9-31. https:// bit.ly/3pcLp4Y

González, M. (2019). Fake News: desinformación en la era de la sociedad de la información. Ámbitos. Revista Internacional de Comunicación. (45), pp. 29-52. http://dx.doi.org/10.12795/Ambitos.2019.i45.03

Llácer, E. y Ballesteros, F. (2012) El lenguaje científico, la divulgación de la ciencia y el riesgo de la pseudociencia. Quaderns de Filologia. Estudis Linguístics. Volumen XVII, pp. 54-67. https://bit. ly/32qXVUT

Marmón, C. (2014). Prácticas discursivas académicas en la economía: aproximación crítica a las estrategias de valoración. Revista de Investigación Lingüística. (17), 75-95. https://bit.ly/2GKuN3o

Ministerio de Educación (2006). Normas para la organización y aplicación del Plan Lector en las instituciones educativas de Educación Básica Regular. https://bit.ly/30DWGRj

Muñoz, E., Muñoz, L, García, M. y Granado, L. (2013). La comprensión lectora de textos científicos en el proceso de enseñanza- aprendizaje. Humanidades Médicas. Volumen 13 (3), pp. 772- 804. https://bit. ly/2U6JyQZ 
Oficina Central de Admisión [OCA] de la Universidad Nacional Mayor de San Marcos [UNMSM]. (15 de setiembre 2020). Examen Ordinario de Admisión 2020-I. https://bit.ly/2HSUAGE

Oficina Central de Admisión [OCA] de la Universidad Nacional Mayor de San Marcos [UNMSM]. (10 de marzo 2019). Examen Ordinario de Admisión 2019. II. https://bit.ly/3d3kKSG

Oficina Central de Admisión [OCA] de la Universidad Nacional Mayor de San Marcos [UNMSM]. (16 de setiembre 2018). Examen Ordinario de Admisión 2019-I. https://bit.ly/36BMz3q
Oficina Central de Admisión [OCA] de la Universidad Nacional Mayor de San Marcos [UNMSM]. (18 de marzo 2018). Examen Ordinario de Admisión 2018. II. https://bit.ly/33y3XUD

Oficina Central de Admisión [OCA] de la Universidad Nacional Mayor de San Marcos [UNMSM]. (17 de setiembre 2017). Examen Ordinario de Admisión 2018-I. https://bit.ly/3d0aR8a

Scimago Institutions Rankings (2020). [Ranking según trabajos de investigación de universidades Latinoamericanas al 2020]. https://bit.ly/2GJRzI3

RIDU / Revista Digital de Investigación en Docencia Universitaria / e-ISSN: 2223-2516

(C) Los autores. Este artículo es publicado por la Revista Digital de Investigación en Docencia Universitaria del Área de Institutional Research and Effectiveness de la Dirección de Aseguramiento de la Calidad, Universidad Peruana de Ciencias Aplicadas. Este es un artículo de acceso abierto, distribuido bajo los términos de la LicenciaCreativeCommons Atribución-CompartirIgual 4.0 Internacional.( http://creativecommons.org/licenses/by-sa/4.0/), que permite el uso no comercial, distribución y reproducción en cualquier medio, siempre que la obra original sea debidamente citada. 